Dedicated to Dragoš Cvetković on the occasion of his 75th birthday

Applicable Analysis and Discrete Mathematics

available online at http://pefmath.etf.rs

Appl. Anal. Discrete Math. x (xxxx), xxx-xxx. doi:10.2298/AADMxxxxxxxx

\title{
RESISTANCE DISTANCES ON NETWORKS
}

\author{
A. Carmona, A.M. Encinas, M. Mitjana
}

This paper aims to study a family of distances in networks associated with effective resistances. Specifically, we consider the effective resistance distance with respect to a positive parameter and a weight on the vertex set; that is, the effective resistance distance associated with an irreducible and symmetric $M$-matrix whose lowest eigenvalue is the parameter and the weight function is the associated eigenfunction. The main idea is to consider the network embedded in a host network with additional edges whose conductances are given in terms of the mentioned parameter. The novelty of these distances is that they take into account not only the influence of shortest and longest weighted paths but also the importance of the vertices. Finally, we prove that the adjusted forest metric introduced by P. Chebotarev and E. Shamis is nothing else but a distance associated with a Schrödinger operator with constant weight.

\section{INTRODUCTION}

Because of its structural meaning, the resistance distance has become a useful tool to analyze structural properties of graphs, or more generally of networks, such as robustness, see for instance [13]. In this context, a network is a connected graph in which each edge has been assigned a positive value, named the conductance of the edge. In contrast with the standard geodesic distance, defined as the length of the shortest path between vertices, the resistance distance takes into account all

2010 Mathematics Subject Classification. 05C12.

Keywords and Phrases. Effective resistances, resistance distances, Schrödinger operator, symmetric $M$-matrices. 
paths between vertices. The high sensibility of this metric with respect to small perturbations, makes it suitable to compare different network structures. This is one of the main reasons for which effective resistances and the corresponding Kirchhoff Index, the sum of all of them, have emerged as a structure-descriptor in the framework of Organic Chemistry, where the topology of chemical compounds is conventionally represented by a molecular network where edge weights correspond to bond properties, see for instance $[\mathbf{1 5}]$ and references therein. Effective resistances can be expressed in terms of the group inverse of the Laplacian matrix.

In this work we associate a positive value to each node of the network, that will be called a weight on the network. Then, we define a one parametric family of resistance distances associated with each weight function. In fact, each distance of this family is defined throughout a positive semi-definite Schrödinger operator on the network where the parameter is the lowest eigenvalue and the weight function is the associated eigenfunction. Therefore, the main properties of these distances can be analyzed within the framework of potential theory. From the matrix point of view, this is equivalent to define the effective resistance associated with an irreducible and symmetric $M$-matrix, see [2].

For a fixed weight function, we prove that the associated effective resistance distances are continuous and monotone decreasing with respect to the parameter and they are upper bounded by the weighted geodesic distance of the network. Moreover, the effective resistance with respect to a parameter and a weight coincides with the weighted geodesic distance iff the parameter is null and the network is a tree. In addition, the resistance distance associated with a positive semi-definite Schrödinger operator is graph geodetic iff the operator is singular, see next section for the definition of graph geodetic distance.

We prove that for each weight function, the corresponding one parametric family of resistance distances can be understood as the effective resistance on a host network associated with a singular positive semi-definite Schrödinger operator. The initial network is embedded in the host network that consists in a new vertex joined with any former vertex by an edge whose conductance is the product of the parameter by the weight. This explains the monotonicity property of the one parametric family in the sense that the larger the parameter the lower the resistance. We illustrate this property through a simple example of a graph with three vertices. Moreover, in the case of constant weight, we apply some electrical equivalences to show that the one-parametric family of effective resistances can be seen the effective resistance associated with the combinatorial Laplacian of a complete network. This property, can be generalized for arbitrary weights by considering an appropriate embedding on the host network, see [6].

For any network with constant weight on the vertices, the one parametric family of distances corresponds to the effective resistance associated with symmetric and diagonally dominant $M$-matrices with constant diagonal excess. We show that these resistance distances coincide with the so-called adjusted forest metrics introduced by P. Chebotarev and E. Shamis at the late 90's; see [8]. They interpret these metrics as a measure of the accessibility from a vertex to another and they 
form a one-parametric family of distances, where the parameter determines the proportion of taking into account long and short routes between vertices.

\section{PRELIMINARIES}

Given a finite set $V$, the set of real valued functions on $V$ is denoted by $\mathcal{C}(V)$. The standard inner product on $\mathcal{C}(V)$ is denoted by $\langle\cdot, \cdot\rangle$ and hence, if $u, v \in \mathcal{C}(V)$, then $\langle u, v\rangle=\sum_{x \in V} u(x) v(x)$. For any $x \in V, \varepsilon_{x} \in \mathcal{C}(V)$ stands for the Dirac function at $x$ and 1 is the function defined by $1(x)=1$, for any $x \in V$. On the other hand, $\omega \in \mathcal{C}(V)$ is called a weight if it satisfies that $\omega(x)>0$ for any $x \in V$ and moreover $\langle\omega, \omega\rangle=n=|V|$. The set of weights on $V$ is denoted by $\Omega(V)$.

The triple $\Gamma=(V, E, c)$ denotes a finite network; that is, a finite connected graph without loops nor multiple edges, with vertex set $V$, whose cardinality equals $n$, and edge set $E$, in which each edge $\{x, y\}$ has been assigned a conductance $c(x, y)>0$. So, the conductance can be considered as a symmetric function $c: V \times$ $V \longrightarrow[0,+\infty)$ such that $c(x, x)=0$ for any $x \in V$ and moreover, vertex $x$ is adjacent to vertex $y$ iff $c(x, y)>0$.

Denote by $P_{x y}=\left\{x=x_{1} \sim x_{2} \sim \cdots \sim x_{k}=y\right\}$ a path joining vertices $x$ and $y$, the length of the weighted path $P_{x y}$ is $\ell_{c}\left(P_{x y}\right)=\sum_{i=1}^{k-1} \frac{1}{c\left(x_{i}, x_{i+1}\right)}$. The geodesic distance between two vertices $x$ and $y$ is defined as the least resistive path; that is,

$$
d_{c}(x, y)=\min \left\{\ell_{c}\left(P_{x y}\right): P_{x y} \text { is a path from } x \text { to } y\right\} .
$$

The function $d_{c}$ determines a distance on the network that fulfills the property that the triangular inequality is an equality when the central node separates the two others; that is, $d_{c}(x, y)=d_{c}(x, z)+d_{c}(z, y)$ if every path from $x$ to $y$ passes through $z$. In general a distance, $d$, on a network is called graph geodetic when $d(x, y)=d(x, z)+d(z, y)$ if and only if every path from $x$ to $y$ passes through $z$. This kind of distances are also called cutpoint additive, see [9]. In general, the geodesic distance is not graph geodetic in the mentioned sense. We point out that some authors used the notion of geodetic distance in a weaker sense, see $[\mathbf{1 2}$, page 278].

The combinatorial Laplacian or simply the Laplacian of the network $\Gamma$ is the endomorphism of $\mathcal{C}(V)$ that assigns to each $u \in \mathcal{C}(V)$ the function

$$
\mathcal{L}(u)(x)=\sum_{y \in V} c(x, y)(u(x)-u(y)), \quad x \in V .
$$

Given $q \in \mathcal{C}(V)$, the Schrödinger operator on $\Gamma$ with potential $q$ is the endomorphism of $\mathcal{C}(V)$ that assigns to each $u \in \mathcal{C}(V)$ the function $\mathcal{L}_{q}(u)=\mathcal{L}(u)+q u$, where $q u \in \mathcal{C}(V)$ is defined as $(q u)(x)=q(x) u(x)$; see for instance $[\mathbf{1}, \mathbf{4}]$. For any 
$\omega \in \Omega(V)$, the potential associated with $\omega$ is $q_{\omega}=-\frac{1}{\omega} \mathcal{L}(\omega)$. It is well-known that any Schrödinger operator is self-adjoint and moreover, it is positive semi-definite iff there exist $\omega \in \Omega(V)$ and $\lambda \geq 0$ such that $q=q_{\omega}+\lambda$; see [1]. In addition, $\mathcal{L}_{q}$ is singular iff $\lambda=0$, in which case $\left\langle\mathcal{L}_{q_{\omega}}(v), v\right\rangle=0$ iff $v=a \omega, a \in \mathbb{R}$. In any case, $\lambda$ is the lowest eigenvalue of $\mathcal{L}_{q}$ and its associated eigenfunctions are multiples of $\omega$.

The connection between positive semi-definite Schrödinger operators and irreducible, symmetric $M$-matrices can be explained as follows. Suppose that $V=\left\{x_{1}, \ldots, x_{n}\right\}$ and consider $c_{i j}=c\left(x_{i}, x_{j}\right)=c_{j i}$. Then, each $u \in \mathcal{C}(V)$ is identified with $\left(u\left(x_{1}\right), \ldots, u\left(x_{n}\right)\right)^{T} \in \mathbb{R}^{n}$ and the combinatorial Laplacian of $\Gamma$ is identified with the irreducible matrix

$$
\mathrm{L}=\left[\begin{array}{cccc}
\kappa_{1} & -c_{12} & \cdots & -c_{1 n} \\
-c_{21} & \kappa_{2} & \cdots & -c_{2 n} \\
\vdots & \vdots & \ddots & \vdots \\
-c_{n 1} & -c_{n 2} & \cdots & \kappa_{n}
\end{array}\right]
$$

where $\kappa_{i}=\sum_{j=1}^{n} c_{i j}, i=1, \ldots, n$. Clearly, this matrix is symmetric and diagonally dominant and hence it is positive semi-definite. Moreover, it is singular and 0 is a simple eigenvalue whose associated eigenvectors are constant. Observe that the matrix associated with a positive semi-definite $\mathcal{L}_{q}$ is

$$
\mathbf{L}_{q}=\left[\begin{array}{cccc}
\kappa_{1}+q\left(x_{1}\right) & -c_{12} & \cdots & -c_{1 n} \\
-c_{12} & \kappa_{2}+q\left(x_{2}\right) & \cdots & -c_{2 n} \\
\vdots & \vdots & \ddots & \vdots \\
-c_{1 n} & -c_{2 n} & \cdots & \kappa_{n}+q\left(x_{n}\right)
\end{array}\right]
$$

which is an irreducible and symmetric $M$-matrix but not necessarily diagonally dominant, see $[\mathbf{1}]$.

If $\mathcal{L}_{q}$ is positive definite, then it is invertible and its inverse is called the Green operator. On the other hand, when $\mathcal{L}_{q}$ is positive semi-definite and singular the operator that assigns to each function $f \in \mathcal{C}(V)$ the unique $u \in \mathcal{C}(V)$ such that $\mathcal{L}_{q}(u)=f-\frac{1}{n}\langle\omega, f\rangle \omega$ and $\langle u, \omega\rangle=0$ is called the Green operator. In any case, the Green operator is denoted by $\mathcal{G}_{q}$, see [2]. Moreover, the function $G_{q}: V \times V \longrightarrow \mathbb{R}$, defined as $G_{q}(x, y)=\mathcal{G}_{q}\left(\varepsilon_{y}\right)(x)$, for any $x, y \in V$, is called the Green function. Observe that $\mathcal{G}_{q}(\omega)=\lambda^{\dagger} \omega$, where $\lambda^{\dagger}=\lambda^{-1}$ when $\lambda>0$ and $\lambda^{\dagger}=0$ when $\lambda=0$. Moreover, $\mathcal{G}_{q}$ is self-adjoint as a consequence of the Fredholm Alternative and $\mathcal{G}_{q}$ is a symmetric function. Observe that, in the singular case, the Green operator is nothing else but the group inverse of $\mathrm{L}_{q}$.

\section{RESISTANCE DISTANCES}


In [2], the authors introduced a generalization of the concept of effective resistance with respect to a value $\lambda \geq 0$ and a weight $\omega \in \Omega(V)$. Specifically, from the functional on $\mathcal{C}(V)$ defined as

$$
\mathfrak{J}_{x, y}(u)=2\left[\frac{u(x)}{\omega(x)}-\frac{u(y)}{\omega(y)}\right]-\left\langle\mathcal{L}_{q}(u), u\right\rangle
$$

where $q=q_{\omega}+\lambda$, we defined the generalization of the effective resistance.

Definition 3.1. Given $x, y \in V$, the effective resistance between $x$ and $y$ with respect to $\lambda$ and $\omega$, is the value

$$
R_{\lambda, \omega}(x, y)=\max _{u \in \mathcal{C}(V)}\left\{\mathfrak{J}_{x, y}(u)\right\}
$$

In the sequel we omit the expression with respect to $\lambda$ and $\omega$ when it does not lead to confusion. When $\lambda=0$ we usually drop the subindex $\lambda$ in the above expressions and when $\omega$ is constant we also omit the subindex $\omega$. Therefore, $R$ is nothing else than the standard effective resistance of the network.

Since the matrix associated with the Schrödinger operator $\mathcal{L}_{q}$ is an irreducible, symmetric $M$-matrix and conversely, each irreducible, symmetric $M-$ matrix appears as associated with a Schrödinger operator, we can assign an effective resistance function to any irreducible, symmetric $M$-matrix. Notice that $\lambda$ is the lowest eigenvalue, both of the matrix and the Schrödinger operator, and $\omega$ is its corresponding eigenfunction. Therefore, our study includes all the irreducible, symmetric $M$-matrices, not necessarily diagonally-dominant.

From now on we consider the function $\tau_{x y}=\omega^{-1}\left(\varepsilon_{x}-\varepsilon_{y}\right)$. The following result can be found in [2] and allows us to express the effective resistances in terms of the solution of a Poisson equation. In particular, these expressions will be useful to prove the main properties of the effective resistances.

Proposition 3.2. If $u \in \mathcal{C}(V)$ is a solution of the Poisson equation $\mathcal{L}_{q}(u)=\tau_{x y}$, then

$$
R_{\lambda, \omega}(x, y)=\left\langle\mathcal{L}_{q}(u), u\right\rangle=\frac{u(x)}{\omega(x)}-\frac{u(y)}{\omega(y)} .
$$

Therefore, $R_{\lambda, \omega}$ is symmetric, non-negative and moreover $R_{\lambda, \omega}(x, y)=0$ iff $x=y$. In addition,

$$
R_{\lambda, \omega}(x, y)=\frac{G_{q}(x, x)}{\omega^{2}(x)}+\frac{G_{q}(y, y)}{\omega^{2}(y)}-\frac{2 G_{q}(x, y)}{\omega(x) \omega(y)} .
$$

Using the so-called Doob transform we can rewrite the expression for the quadratic part of the functional that defines the effective resistance, in the following way:

$$
\left\langle\mathcal{L}_{q}(u), u\right\rangle=\sum_{x, y \in V} c(x, y) \omega(x) \omega(y)\left(\frac{u(x)}{\omega(x)}-\frac{u(y)}{\omega(y)}\right)^{2}+\lambda \sum_{x \in V} u^{2}(x) .
$$


If $0=\lambda_{1}<\lambda_{2} \leq \cdots \leq \lambda_{n}$ are the eigenvalues of $\mathcal{L}_{q_{\omega}}$ and $\left\{u_{j}\right\}_{j=1}^{n} \subset \mathcal{C}(V)$ is an orthonormal basis of eigenfunctions, where $u_{1}=\frac{1}{\sqrt{n}} \omega$, then

$$
G_{q_{\omega}}(v)=\sum_{i=2}^{n} \frac{1}{\lambda_{i}}\left\langle v, u_{i}\right\rangle u_{i} .
$$

Moreover, for $\lambda>0$, the eigenvalues of $\mathcal{L}_{q}$ are $\lambda=\lambda+\lambda_{1}<\lambda+\lambda_{2} \leq \cdots \leq \lambda+\lambda_{n}$, whereas the eigenfunctions are the same. Hence, for $q=q_{\omega}+\lambda$

$$
G_{q}(v)=\frac{1}{n \lambda}\langle\omega, v\rangle \omega+\sum_{i=2}^{n} \frac{1}{\lambda_{i}+\lambda}\left\langle v, u_{i}\right\rangle u_{i} .
$$

From this expression we get the following result whose proof can be found in [3].

Proposition 3.3. [3, Proposition 3.4] For any $x, y \in V$ it is verified that

$$
R_{\lambda, \omega}(x, y)=\sum_{i=2}^{n} \frac{n}{\lambda+\lambda_{i}}\left(\frac{u_{i}(x)}{\omega(x)}-\frac{u_{i}(y)}{\omega(y)}\right)^{2} .
$$

The next result contains the main properties of the effective resistances associated with Schrödinger operators.

Theorem 3.4. If $\Gamma$ is a connected network, the effective resistance with respect to a parameter and a weight satisfies the following properties:

(i) The effective resistance $R_{\lambda, \omega}$ determines a distance on the network. Moreover, $R_{\lambda, \omega}(x, y)=R_{\lambda, \omega}(x, z)+R_{\lambda, \omega}(z, y)$ iff $\lambda=0$ and $z$ separates $x$ and $y$.

(ii) If $0 \leq \hat{\lambda} \leq \lambda$ and $\hat{q}=q_{\omega}+\hat{\lambda}$, then $R_{\lambda, \omega} \leq R_{\hat{\lambda}, \omega} \leq R_{\omega}$.

(iii) $R_{\lambda, \omega}(x, y) \leq d_{\hat{c}}(x, y)$, where $\hat{c}(x, y)=c(x, y) \omega(x) \omega(y)$, with equality iff $\lambda=0$ and there exists a unique path from $x$ to $y$.

(iv) $\lim _{\lambda \rightarrow+\infty} R_{\lambda, \omega}=0$ and $\lim _{\lambda \rightarrow 0} R_{\lambda, \omega}=R_{\omega}$.

Proof. (i) The proof can be found in [2, Corollary 4.4].

(ii) It is enough to keep in mind the expression for the effective resistance given in equation (3).

(iii) Let us firstly show that if $x \sim y$; i.e., $c(x, y)>0$ then $R_{\omega}(x, y) \leq \frac{1}{\hat{c}(x, y)}$. Let $u$ be a solution of the Poisson equation $\mathcal{L}_{q_{\omega}}(u)=\tau_{x y}$, then

$$
\begin{aligned}
R_{\omega}(x, y) & =\frac{u(x)}{\omega(x)}-\frac{u(y)}{\omega(y)}=\left\langle\mathcal{L}_{q_{\omega}}(u), u\right\rangle \\
& =\sum_{z \sim t} \hat{c}(z, t)\left(\frac{u(z)}{\omega(z)}-\frac{u(t)}{\omega(t)}\right)^{2} \\
& \geq \hat{c}(x, y)\left(\frac{u(x)}{\omega(x)}-\frac{u(y)}{\omega(y)}\right)^{2}=\hat{c}(x, y) R_{\omega}(x, y)^{2} .
\end{aligned}
$$


Keeping in mind that $R_{\omega}(x, y) \neq 0$, we get that

$$
R_{\omega}(x, y) \leq \frac{1}{\hat{c}(x, y)}
$$

Moreover, $R_{\omega}(x, y)=\frac{1}{\hat{c}(x, y)}$ iff $\frac{u(z)}{\omega(z)}$ is constant in each connected component of $\Gamma \backslash\left\{e_{x y}\right\}$. Therefore, $x$ and $y$ cannot be in the same connected component, since otherwise $\frac{u(x)}{\omega(x)}=\frac{u(y)}{\omega(y)}$ and hence $R_{\omega}(x, y)=0$. In this case, $R_{\omega}(x, y)=\frac{1}{\hat{c}(x, y)}=$ $d_{\hat{c}}(x, y)$ iff there exists a unique weighted path from $x$ to $y$.

Consider now $x, y \in V$ arbitrary vertices. From (ii), it is enough to prove that $R_{\omega}(x, y) \leq d_{\hat{c}}(x, y)$. Let $P_{x y}=\left\{x=x_{1} \sim x_{2} \sim \cdots \sim x_{k}=y, x_{i} \in V\right\}$ be a shortest weighted path from $x$ to $y$. Then, from the triangular inequality and the case of adjacent vertices we get that

$$
R_{\omega}(x, y) \leq R_{\omega}\left(x, x_{1}\right)+\cdots+R_{\omega}\left(x_{k-1}, y\right) \leq \frac{1}{\hat{c}\left(x, x_{1}\right)}+\cdots+\frac{1}{\hat{c}\left(x_{k-1}, y\right)}=d_{\hat{c}}(x, y) \text {. }
$$

In particular, $R_{\omega}(x, y)=d_{\hat{c}}(x, y)$ iff any of the above inequality are equalities; i.e., there exists a unique path from $x$ to $y$. Moreover, from (3) we also obtain $R_{\lambda, \omega}(x, y)=d_{\hat{c}}(x, y)$ if in addition $\lambda=0$.

(iv) From Proposition 3.3, for any $x, y \in V$,

$$
R_{\lambda, \omega}(x, y)=\sum_{i=2}^{n} \frac{n}{\lambda+\lambda_{i}}\left(\frac{u_{i}(x)}{\omega(x)}-\frac{u_{i}(y)}{\omega(y)}\right)^{2}
$$

Therefore,

$$
\lim _{\lambda \rightarrow+\infty} R_{\lambda, \omega}(x, y)=0 \text { and } \lim _{\lambda \rightarrow 0} R_{\lambda, \omega}(x, y)=R_{\omega}(x, y)
$$

Observe that property (i) means that the effective resistances with respect to a weight and a parameter are graph geodetic iff $\lambda=0$. Moreover for fixed weight, $\omega$, we can define a one-parametric family of distances given by $R_{\lambda, \omega}$. This family is continuous and monotone decreasing with respect to $\lambda$.

The interpretation of the effective resistance with respect to a non-negative parameter and a weight was raised in [2], through a commonly used technique in the context of electrical networks and Markov chains, see $[\mathbf{1 0}, \mathbf{1 1}]$. The goal was to generalize the Fiedler characterization of irreducible, symmetric and diagonally dominant $M$-matrices as resistive inverses, see [14], to all irreducible and symmetric $M$-matrices or equivalently, to all positive semi-definite Schrödinger operators.

Given a positive-definite Schrödinger operator on $\Gamma, \mathcal{L}_{q}$, the method consists in embedding the given network into a suitable host network. The new network is constructed by adding a new vertex, that represents a grounded vertex or an absorbing state in the context of Markov chains. The new vertex is joined with each vertex in the original network through a new edge whose conductance is the diagonal 


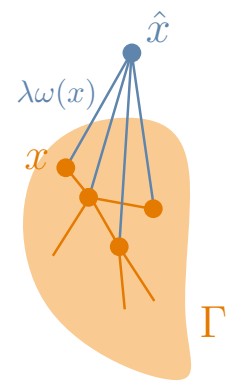

Figure 1: Host network

excess of the original $M$-matrix after the application of the Doob transform; see Figure 1.

Given $\lambda>0, \omega \in \Omega(V)$ and $\hat{x} \notin V$, we consider the network $\Gamma_{\lambda, \omega}=(V \cup$ $\left.\{\hat{x}\}, c_{\lambda, \omega}\right)$ where $c_{\lambda, \omega}(x, y)=c(x, y)$ when $x, y \in V$ and $c_{\lambda, \omega}(\hat{x}, x)=c_{\lambda, \omega}(x, \hat{x})=$ $\lambda \omega(x)$ for any $x \in V$. We denote by $\mathcal{L}^{\lambda, \omega}$ its combinatorial Laplacian and by $\widehat{\omega} \in \Omega(V \cup\{\hat{x}\})$ the weight given by $\widehat{\omega}(x)=\omega(x)$ when $x \in V$ and $\widehat{\omega}(\hat{x})=1$.

In $[\mathbf{2}$, Corollary 4.6$]$ it was proved that the effective resistance with respect to a parameter and a weight equals the effective resistance in the host graph for the associated singular operator; that is,

$$
R_{\lambda, \omega}(x, y)=\widehat{R}_{\hat{\omega}}(x, y) .
$$

This interpretation confirms the monotonicity property of the resistance distances, since the larger the parameter the lower the resistance.

We illustrate the procedure with a small example. Let $\Gamma$ be the path on three vertices is $V=\left\{x_{1}, x_{2}, x_{3}\right\}$ whose non null conductances are $c\left(x_{1}, x_{2}\right)=c_{1}$ and $c\left(x_{2}, x_{3}\right)=c_{2}$. We consider its host network $\Gamma_{\lambda, \omega}=\left(\left\{x_{1}, x_{2}, x_{3}\right\} \cup\{\hat{x}\}, c_{\lambda, \omega}\right)$ as defined above. We set $c_{\lambda, \omega}\left(x_{i}, x_{j}\right)=c\left(x_{i}, x_{j}\right)$ when $i, j=1,2,3$ and $c_{\lambda, \omega}\left(\hat{x}, x_{i}\right)=$ $\lambda \omega\left(x_{i}\right)$ for any $i=1,2,3$. Then computing the group inverse of $\mathcal{L}^{\lambda, \omega}$ and using Proposition 3.2 we get that

$$
\begin{aligned}
& \widehat{R}_{\hat{\omega}}\left(x_{1}, x_{2}\right)=\frac{\lambda \omega_{3}\left(\omega_{1}^{2}+\omega_{2}^{2}\right)+3 c_{2} \omega_{2}}{\omega_{1} \omega_{2} \omega_{3}\left(c_{1} \lambda \omega_{1}^{2}+c_{1} \lambda \omega_{2}^{2}+\lambda^{2} \omega_{1} \omega_{2}\right)+\omega_{1} \omega_{2} c_{2}\left(3 c_{1} \omega_{2}+3 \lambda \omega_{1}-\lambda \omega_{1}^{3}\right)} \\
& \widehat{R}_{\hat{\omega}}\left(x_{2}, x_{3}\right)=\frac{\lambda \omega_{1}\left(\omega_{2}^{2}+\omega_{3}^{2}\right)+3 c_{1} \omega_{2}}{\omega_{1} \omega_{2} \omega_{3}\left(c_{2} \lambda \omega_{3}^{2}+c_{2} \lambda \omega_{2}^{2}+\lambda^{2} \omega_{2} \omega_{3}\right)+\omega_{2} \omega_{3} c_{1}\left(3 c_{2} \omega_{2}+3 \lambda \omega_{3}-\lambda \omega_{3}^{3}\right)} \\
& \widehat{R}_{\hat{\omega}}\left(x_{1}, x_{3}\right)=\frac{\lambda \omega_{2}\left(\omega_{1}^{2}+\omega_{3}^{2}\right)+3 c_{1} \omega_{1}+3 c_{2} \omega_{3}}{\omega_{1} \omega_{2} \omega_{3}\left(\lambda^{2} \omega_{1} \omega_{3}+3 c_{1} c_{2}\right)+\omega_{1} \omega_{3} \lambda\left(3 c_{1} \omega_{3}+3 c_{2} \omega_{1}-c_{1} \omega_{3}^{3}-c_{2} \omega_{1}^{3}\right)} .
\end{aligned}
$$

This result coincides with the one obtained in [5, Corollary 5.5] except for a multiplicative constant due to the normalization factor of the weight. 


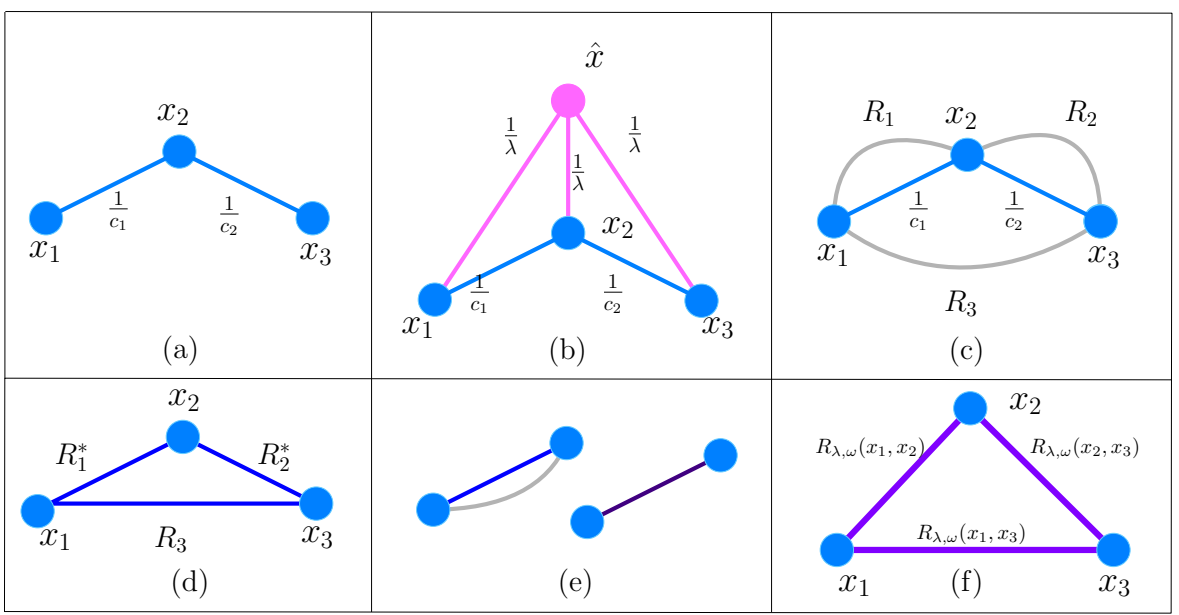

Figure 2: Interpreting the resistance $R_{\lambda, \omega}$

We consider now the above case with $\omega\left(x_{i}\right)=1$ for any $i=1,2,3$. We interpret the effective resistance of the path, Fig 2(a), with respect to a non negative parameter, $\lambda$, and a weight $\omega$, using the host network, Fig 2(b). We have written the inverse of the conductance of each edge, since we are interested in the resistances of the network. From the $\Delta$-Star transform we obtain the network in Fig 2(c) whose new (gray) resistances are

$$
R_{1}=R_{2}=R_{3}=\frac{3}{\lambda}
$$

Next, using parallel transformation, we get an electrically equivalent network, Fig $2(\mathrm{~d})$, with resistance

$$
R_{1}^{*}=\left(\frac{\lambda}{3}+c_{1}\right)^{-1} \quad \text { and } \quad R_{2}^{*}=\left(\frac{\lambda}{3}+c_{2}\right)^{-1} .
$$

This can be seen as a complete network whose conductances have been increased by $\frac{\lambda}{3}$. Again, after series, Fig 2(e), and parallel, Fig 2(f), transforms we get

$$
\begin{aligned}
& R_{\lambda, \omega}\left(x_{1}, x_{2}\right)=\frac{2 \lambda+3 c_{2}}{3 c_{1} c_{2}+2 c_{1} \lambda+2 c_{2} \lambda+\lambda^{2}}, \\
& R_{\lambda, \omega}\left(x_{1}, x_{3}\right)=\frac{2 \lambda+3 c_{1}+3 c_{2}}{3 c_{1} c_{2}+2 c_{1} \lambda+2 c_{2} \lambda+\lambda^{2}}, \\
& R_{\lambda, \omega}\left(x_{2}, x_{3}\right)=\frac{2 \lambda+3 c_{1}}{3 c_{1} c_{2}+2 c_{1} \lambda+2 c_{2} \lambda+\lambda^{2}} .
\end{aligned}
$$

Finally, let us consider the particular case in which $\omega$ is the constant weight and $\lambda>0$ in an arbitrary network. In $[\mathbf{7}, \mathbf{8}]$ Chebotarev et. al. introduced two 
parametric families of distances in a network: the so-called forest distance between $x$ and $y$ and the adjusted forest distance between $x$ and $y$. Both families are defined by emulating the expression for the classical effective resistance in terms of the inverse of the combinatorial Laplacian. Our purpose now is to show that these families are nothing else but the effective resistances with respect to a parameter $\lambda>0$, and hence all the properties can be directly obtained.

In this case, the associated Schrödinger operator will be $\mathcal{L}_{\lambda}=\mathcal{L}+\lambda I$. Denote by $G_{\lambda}$ the Green function associated with $\mathcal{L}_{\lambda}$. As this operator is invertible we know that $G_{\lambda}=\mathcal{L}_{\lambda}^{-1}$.

In $[\mathbf{7}, \mathbf{8}]$ the authors introduced the matrices $Q_{\alpha}$, for a parameter $\alpha>0$, where

$$
Q_{\alpha}=(I+\alpha \mathcal{L})^{-1} .
$$

The parameter $\alpha$ determines the proportion of considering long and short walks between vertices. Using this matrix they introduced the mentioned forest distance, $d^{\alpha}$, and adjusted forest distance, $\rho^{\alpha}$, between $i$ and $j$ defined as the values

$$
d_{i j}^{\alpha}=\frac{1}{2}\left(q_{i i}^{\alpha}+q_{j j}^{\alpha}-q_{i j}^{\alpha}-q_{j i}^{\alpha}\right), \quad \rho_{i j}^{\alpha}=\alpha\left(q_{i i}^{\alpha}+q_{j j}^{\alpha}-q_{i j}^{\alpha}-q_{j i}^{\alpha}\right),
$$

once the set of vertices $V$ has been labeled. Observe that, if we take $\lambda=\alpha^{-1}$, then

$$
\mathcal{L}_{\lambda}=(\mathcal{L}+\lambda I)=\alpha^{-1}(\alpha \mathcal{L}+I)
$$

and hence

$$
Q_{\alpha}=\lambda G_{\lambda}
$$

Therefore,

$$
d_{i j}^{\alpha}=\frac{\lambda}{2} R_{\lambda}(i, j) \text { and } \rho_{i j}^{\alpha}=R_{\lambda}(i, j) .
$$

This relation explains the factor $\frac{1}{\alpha}$ introduced by Chebotarev et al. for the adjusted forest metric. The authors realized that for the forest distance $\lim _{\alpha \rightarrow \infty} d_{i j}^{\alpha}=0$, which has no sense, whereas $\lim _{\alpha \rightarrow \infty} \rho_{i j}^{\alpha}=R(i, j)$. In [8], the authors introduced the notion of $\alpha$-extension that is similar to the notion of host graph, in order to prove that the adjusted forest metric is the resistance distance for the combinatorial Laplacian of the $\alpha$-extension.

The generalized effective resistance distances are distances that take into account not only the proportion of short and long path between $x$ and $y$ but also the importance of the vertices $x$ and $y$ in the network, read in terms of the weight $\omega$.

\section{Acknowledgments}

We are grateful to the reviewers for taking the time to provide quite valuable comments and suggestions.

This work has been partly supported by the Spanish Research Council (Comisión Interministerial de Ciencia y Tecnología,) under project MTM2014-60450-R. 


\section{REFERENCES}

1. E. Bendito, A. Carmona and A. M. Encinas. Potential theory for Schrödinger operators on finite networks, Rev. Mat. Iberoamericana 21 (2005) 771-818.

2. E. Bendito, A. Carmona, A.M. Encinas and J.M. Gesto. Characterization of symmetric $M$-matrices as resistive inverses. Linear Algebra Appl., 430 (2009) 1336-1349.

3. E. Bendito, A. Carmona, A.M. Encinas, J.M. Gesto and M. Mitjana. The Kirchhoff indices of join networks. in Linear Algebra Appl., 432 (2010) 2278-2292.

4. T. Biyikoğlu, J. Leydold and P.F. Stadler. Laplacian Eigenvectors of Graphs, LNM 1915, Springer, Berlin, 2007.

5. A. Carmona, A.M. Encinas and M. Mitjana. Discrete elliptic operators and their Green operators. Linear Algebra Appl., 442 (2014) 115-134.

6. A. Carmona, A.M. Encinas and M. Mitjana. Star-mesh transforms on networks. submitted.

7. P. Chebotarev and E. Shamis. On proximity measures for graphs vertices. Automat. Remote Control, 59 (1998) 1443-1459.

8. P. Chebotarev and E. Shamis. The Forest Metrics for Graph Vertices. Electron. Notes Discrete Math., 11 (2002) 98-107.

9. P. Chebotarev. Studying New Classes of Graph Metrics, in Geometric science of information. Lecture Notes in Comput. Sci., 8085, Springer, Heidelberg, (2013) 207214.

10. C. Dellacherie, S. Martínez and J. San Martín. Ultrametric matrices and induced Markov Chains, Adv. Appl. Math., 17 (1996) 169-183.

11. C. Dellacherie, S. Martínez and J. San Martín. Description of the sub-Markov kernel associated to generalized ultrametric matrices. An algorithm approach, Linear Algebra Appl., 318 (2000) 1-21.

12. M.M. Deza and E. Deza. Encyclopedia of distances (third edition). Springer, Heidelberg, 2014.

13. W. Ellens, F.M. Spieksma, P. Van Mieghem, A. Jamakovic, R.E. Kooij. Effective graph resistance, Linear Algebra Appl. 435 (2011) 2491-2506.

14. M. Fiedler. Some characterizations of symmetric $M$-matrices, Linear Algebra Appl. 275-276 (1998) 179-187.

15. Y. Yang and D.J. Klein. A recursion formula for resistance distances and its applications, Discrete Appl. Math. 161 (2013) 2702-2715. 